\title{
COVID-19 EN IBEROAMÉRICA: UN ACERCAMIENTO DESDE LA DERMATOLOGÍA
}

\author{
María González-Payares ${ }^{1 *}$ \\ ${ }^{1}$ Universidad del Norte, Barranquilla, Colombia \\ *Autor de correspondencia: mdpayares@uninorte.edu.co
}

Recibido Octubre 2020; Aceptado Diciembre 2020

\begin{abstract}
Resumen - El COVID-19 fue predominantemente más prevalente entre adultos mayores de 15 años en las primeras etapas del brote y la proporción de casos confirmados entre niños fue relativamente menor. Sin embargo, debido a la creciente propagación mundial del SARS-CoV-2, tenemos nuevos desafíos para la prevención y el control de la epidemia de COVID-19 entre la piel y las manifestaciones cutáneas del virus. Se realizó una revisión bibliográfica no sistemática basado en búsqueda de artículos utilizando las palabras de filtro "Dermatología, Piel, SARS-Cov-2 y COVID-19”, en las bases de datos electrónicas Redalyc, Dialnet, Google Académico y Scielo. Se establece que no hay suficientes evidencias para asociar las afectaciones en la piel y el COVID-19.
\end{abstract}

Palabras clave: Coronavirus, Covid-19, Dermatología, Pandemia, SARS-CoV-2.

\section{COVID-19 IN IBEROAMERICA: AN APPROACH FROM DERMATOLOGY}

\begin{abstract}
COVID-19 was predominantly more prevalent among adults over 15 years of age in the early stages of the outbreak, and the proportion of confirmed cases among children was relatively lower. However, due to the increasing worldwide spread of SARS-CoV-2, we have new challenges for the prevention and control of the COVID-19 epidemic between the skin and the cutaneous manifestations of the virus. A non-systematic bibliographic review based on a search for articles was carried out using the filter words "Dermatology, Skin, SARS-Cov-2 and COVID-19", in the electronic databases Redalyc, Dialnet, Google Academic and Scielo. that there is not enough evidence to associate skin damage and COVID-19.
\end{abstract}

Keywords: Coronavirus, Covid-19, Dermatology, Pandemic, SARS-CoV-2.

\section{Introducción}

Los coronavirus es una amplia familia de virus RNA (39 especies) que afectan a mamíferos y aves y capaces de producir enfermedades en humanos que van desde el resfrió común (10\%) 
hasta enfermedades severas (Padilla et al., 2020). Hasta la fecha se han reconocidos6 virus como agentes causantes de una gama de infecciones de las vías respiratorias, incluidos $\mathrm{HCoV}$-229E, HCoV-OC43, HCoV-NL63, HCoV-HKU1, SARSCoV (síndrome respiratorio agudo severo) y MERS-CoV (Síndrome Respiratorio del Medio Oriente) (Tapia et al., 2020).

El SARS-CoV se describió en 2003 en una epidemia única en China, que causó más de 700 muertos con un 20-30\% precisando ventilación mecánica y con una letalidad del $10 \%$, especialmente elevada en pacientes con comorbilidades (Oña et al., 2020). El MERS-CoV se detectó por primera vez en 2012 originando un cuadro clínico similar, pero con una mayor letalidad (36\%). Esta infección no se ha extinguido y persisten casos esporádicos. Ambas son zoonosis transmitidas al hombre, la primera a través de murciélagos y la segunda originada en dromedarios (Serrano et al., 2020).

El 31 de diciembre de 2019, 27 casos de neumonía de etiología desconocida fueron notificados en Wuhan, provincia de Hubei, China. La enfermedad, conocida a partir del 12 de enero de 2020 como COVID-19, era producida por un nuevo coronavirus que, a partir del 11 de febrero recibiría el nombre de SARS-Cov-2(3) y se ha propagado por todo el planeta amenazando la estabilidad global no solo por el número de infecciones, enfermos y muertes, sino también por el daño psicológico y económico que produce (Salas et al., 2020).

La emergencia sanitaria, reconocida ya como una pandemia, tuvo su punto de inflexión cuando el 23 de febrero de 2020 la ciudad de Wuhan -donde fueron notificados los primeros casos- fue declarada en cuarentena (Calvo et al., 2020). El 28 de junio de 2020 a las 11 pm., 216 países notificaban la presencia del virus, el número de casos en el planeta se estimaba en 9825539 y las muertes en 495388.

El epicentro de la pandemia, inicialmente en China, se posicionó luego en Europa fundamentalmente en Italia y España- y hoy se localiza en los Estados Unidos de América donde la cifra de infectados crece aceleradamente amenazando con colapsar la infraestructura de salud de ese país. La tendencia actual sugiere su desplazamiento hacia América del sur y África (Osuna et al., 2020).

El virus SARS-CoV-2 es muy contagioso y se transmite rápidamente de persona a persona a través de la tos o secreciones respiratorias, y por contactos cercanos; las gotas respiratorias de más de cinco micras, son capaces de transmitirse a una distancia de hasta dos metros, y las manos o los fómites contaminados con estás secreciones seguido del contacto con la mucosa de la boca, nariz u ojos (Maguiña et al., 2020).

En este sentido, la piel, incluyendo las mucosas, es un órgano que con mucha frecuencia presenta infecciones virales. Estas infecciones pueden estar localizadas primariamente en la piel o manifestarse a nivel cutáneo-mucoso como parte de un cuadro general. Es importante describir que en el último mes ha surgido un creciente interés en el estudio de la aparición de manifestaciones cutáneas en pacientes infectados por COVID-19 ya que se ha hipotetizado que si el virus es el causante de la aparición de manifestaciones cutáneas o es simplemente una casualidad (Herrera et al., 2020). 
Las manifestaciones cutáneas evaluadas en esta recopilación de artículos se identifica patrones tipo: erupción eritematosa, lesiones parecidas a la varicela - vesículas, maculas eritemato purpúricas milimétricas en las flexuras a nivel periaxilar, placas purpúricas, urticaria, exantema morbiliforme, pápulas rojo púrpura en los dedos, lesiones isquémicas acrales, pérdida de cabello masculino “alopecia androgénica” (Carrascosa et al., 2020; Morey et al., 2020).

No existe una clasificación detallada previa ni una descripción de las manifestaciones cutáneas de COVID-19 (Cañete et al., 2020). Esta información puede resultar útil para poder reconocer pacientes sintomáticos y proporcionar información para realizar una investigación más grande y representativa, también podría ser útil para el control epidemiológico, especialmente en áreas donde las pruebas de diagnóstico son escasas (Echeverría et al., 2020).

Por todas estas razones, Es necesario revisar la literatura asociada a las manifestaciones en la piel que puede ocasionar el COVID-19, además, en el contexto de una pandemia y con el poco conocimiento sobre este virus en el ámbito iberoamericano.

\section{Materiales y Métodos}

Se desarrolló una revisión bibliográfica en el periodo de tiempo comprendido entre los meses de marzo a septiembre de 2020 con los términos: Dermatología, Piel, SARS-Cov-2 y COVID-19". Se hicieron búsquedas en las bases de datos electrónicas Redalyc, Dialnet, Google Académico y Scielo. La búsqueda fue restringida por idioma o país de origen de las publicaciones en el contexto iberoamericano. Se tuvieron en cuenta todos los estudios primarios o revisiones bibliográficas tenian como foco de estudio SARS-Cov-2 y COVID-19.

\section{Resultados y Discusión}

Esta es la primera pandemia que ocurre en pleno auge de las tecnologías de información y comunicación, donde la variedad de medios y recursos digitales de fácil acceso pueden generar un efecto paradójico de desinformación y conductas desacertadas. De ahí la importancia del rol activo de los dermatólogos y las sociedades científicas en la difusión de información adecuada (Zimman et al., 2020).

Destacamos que el primer reflejo de los dermatólogos no fue suspender en forma sistemática los tratamientos inmunomoduladores/inmunosupresores, sino evaluar el riesgo-beneficio en cada caso. La suspensión masiva podría conducir a brotes severos con requerimiento de internación, en un momento de escasez relativa de camas (Martin et al., 2020).

Recientemente se comenzaron a describir las manifestaciones cutáneas en relación con la COVID-19, con similitud clínica entre niños y adultos. Hasta el momento se han descrito 5 patrones cutáneos más frecuentes en la enfermedad, ellos son: erupción máculo-papular (47 \%), urticaria (19\%), lesiones acrales tipo perniciosas (19 \%), erupción vesicular (9 \%) y raramente livedo reticularis (6\%). 
La incidencia de las manifestaciones cutáneas en relación con la COVID-19 aún no está bien establecida, la mayoría de las publicaciones solamente se han referido a reportes o series de pocos casos, solamente en España se ha conducido un estudio más amplio con 375 casos (Pérez et al., 2020).

Seguidamente, se expondrán algunos estudios en el contexto iberoamericano que se acercan la realidad en el ámbito de la dermatología y el COVID-19, se inicia con el realizado en Cuba por Granados et al. (2020), donde de la población atendida en la provincia de Villa Clara, el 23,8 \% de los pacientes presentó manifestaciones cutáneas, cifra similar a la referida por los italianos en un estudio realizado en Lombardía donde determinaron que el 20,4 \% de los pacientes presenta lesiones en piel, y es a su vez una cifra totalmente diferente a la registrada en China en un estudio de cohorte, en el cual solamente el 0,2 \% de los pacientes tuvo afecciones cutáneas.

Por su parte, en España se agregaron otras 2 formas, para un total de 5, que incluían las 3 primeras descritas previamente y además el livedo o necrosis y el eritema-edema acral con algunas vesículas o pústulas descritas como pseudo-chilblain. Los pacientes de esta serie presentaron solamente 2 tipos de lesiones: las eritemato-papulosas en $80 \%$, cifra similar a la encontrada en Italia, pero mayor a la referida por los españoles y las urticariformes en $20 \%$, que ocupan el segundo lugar en frecuencia de aparición (Calvache et al., 2020).

Al igual, Borges et al. (2020), en Ecuador desarrollo un estudio experimental y observó expresión cutánea con los siguientes patrones: erupción purpurica bilateral axilar, erupción maculopapular y erupción urticariforme en tronco y miembros, eritema multiforme en palmas y tronco, erosivo en mucosa y maculas violáceas en manos y pies, patrón pseudo vesicular generalizado que fueron catalogados como síntomas sospechosos de infección por SARS-CoV-2 según criterio clínico.

Las afectaciones de la COVID-19 serían muy variadas e inespecíficas, y podrían no tener relación con la gravedad del cuadro y resolverse de manera espontánea. Entre ellas se han descrito: exantema (eritematoso, petequial, morbiliforme) generalizado o localizado, urticaria generalizada, vesículas variceliformes, lesiones acro-isquémicas (gangrena seca, ampollas y cianosis), lesiones livedoides y lesiones tipo sabañón (Martínez et al., 2020).

En los pacientes con COVID-19 puede producirse un agravamiento de las lesiones cutáneas previas y es posible la aparición de reacciones alérgicas a los tratamientos empleados. Pero recientemente están apareciendo publicaciones de lesiones en la piel que podrían corresponderse con manifestaciones cutáneas del SARSCoV-2 (Padilla et al., 2020).

Además, coincidiendo con la actual pandemia de COVID-19 los dermatólogos alertan de que se están viendo un elevado número de pacientes con lesiones que habitualmente son menos frecuentes y se han comunicado varios casos (no publicados) de niños y jóvenes con lesiones acras, principalmente en los dedos de los pies, (eritematovioláceas, con costras negruzcas, tipo sabañón [eritema pernio]) que se resuelven de modo espontáneo y en ocasiones no se acompañan de otra clínica. 
Aunque dos de ellos resultaron positivos para las muestras de SARS-CoV-2 y otros estuvieron en contacto con sujetos con síntomas respiratorios, se desconoce la historia familiar de la mayoría de estos casos y no es habitual que se les haya hecho pruebas de diagnóstico para este virus (Pérez-García et al., 2020).

En este marco uno de los nuevos síntomas asociados con el COVID-19 y de reciente aparición es la urticaria. La urticaria es una reacción vascular de la piel que se caracteriza por eritema y formación de ronchas debido al incremento localizado de la permeabilidad vascular (VeintimillaQuintana et al., 2020). Los problemas cutáneos iban desde exantema eritematoso (en 14 pacientes), urticaria generalizada (en 3 pacientes) y vesículas tipo varicela (un paciente). La zona del tronco fue la parte más afectada.

El picor era leve o ausente y las lesiones se curaron en pocos días. En el contexto de la pandemia del COVID-19, y al ser la piel un órgano vivo con capacidad de regenerarse, impermeable, resistente y flexible y al ser un órgano vital para el cuerpo humano que se anticipa a numerosas enfermedades, debe ponerse atención a estos síntomas, atípicos del COVID-19, y examinarla cumpliendo las medidas de bioseguridad aun cuando no haya síntomas respiratorios (Macías et al., 2020).

A partir de las necesidades se crean oportunidades, por lo cual debido a las consultas que se están recibiendo sobre lesiones cutáneas asociadas a síntomas de COVID-19, en España se ha puesto en marcha el estudio "COVID-Piel" que recogerá datos a nivel nacional para poder "categorizar la semiología cutánea y analizar su valor diagnóstico y pronóstico” (Romaní et al., 2020).

En síntesis, se detallan las variadas manifestaciones cutáneas, antes mencionadas:

- Erupción cutánea y petequias. La infección por COVID-19 podría presentar con una erupción cutánea y petequias. Durante el brote de COVID-19 en China, estudios clínicos obviamente no se centraron en las manifestaciones cutáneas de COVID-19. Sin embargo, es probable haya existido. La falta de consulta especializada por Dermatología pudo limitar que se haya podido relacionar más casos y poder ampliar la información y descripción precisa de las lesiones.

- Erupción eritematosa, urticaria y lesiones parecidas a la varicela (vesículas). Recientemente, Recalcati et al. (2020) informaron las manifestaciones cutáneas de la infección por COVID-19 en Italia, y describió 3 patrones principales: erupción eritematosa, urticaria y lesiones parecidas a la varicela (vesículas).

- Placas purpúricas. Otro estudio de Cuahe y colaboradores mostró un cuadro clínico de erupción cutánea en la axila de un paciente. Como una nueva infección viral emergente, la presentación dermatológica es interesante característica clínica. Sin embargo, también existe la posibilidad que podría haber otras formas de erupción cutánea con COVID-19. En un informe anterior sobre clásica infección por coronavirus, la erupción puede ser recurrente y las "placas purpúricas" son clínicas. En el caso clínico de Cuahe et al., La erupción podría ser coincidencia o una erupción cutánea inducida por COVID-19 real (Rebollar, 2020). 
- Petequias. Mientras que Joob \& Wiwanitkit reportaron un caso de COVID-19 que inicialmente presentó una erupción cutánea petequial. Las características patológicas de COVID19 se evaluaron mediante autopsias mínimamente invasivas de pulmón, vasos sanguíneos, piel y otros órganos. Degeneración y necrosis del parénquima, se observó la formación de trombos hialinos en pequeños vasos en los pulmones, así como otros órganos.

Esta podría ser la patología subyacente en estos casos y también podría explicar la agrupación de casos de isquemia acral notificados en Italia durante la línea de tiempo del brote de COVID-19. El autor reportó unas pocas docenas de casos, inicialmente presentaron 8 manifestaciones cutáneas como pápulas rojo púrpuricas en pies y manos que evolucionaron hacia ampollas hemorrágicas o desarrollaron una costra negruzca. Sin embargo, las pruebas de laboratorio del caso informado no estaban disponibles, excepto dos de los casos que se confirmaron COVID-19 por RT-PCR, presentando lesiones cutáneas.

Hunts \& Koziatek reportaron un caso de COVID-19 que presentó pápulas rojo púrpuricas en áreas acrales en un asintótico RTPCR confirmó paciente COVID-19. Las lesiones isquémicas acrales pueden ser una presentación rara de un COVID-19 sintomático, La posibilidad debe tenerse en cuenta. Se ha informado una erupción cutánea en 2 de 1.099 pacientes que se presentan con la enfermedad por Coronavirus 2019 en China, como en 14 de 48 pacientes con la misma enfermedad en Italia, pero desafortunadamente sin más Descripción de su semiología.

Los síntomas están surgiendo particularmente fuera de la esfera respiratoria, como la anosmia y la ageusia, que son síntomas publicados recientemente por Otorrinolaringología. Sobre la manifestación de la piel, pocos casos de erupciones en pacientes con COVID-19 confirmado por laboratorio se describió en dos cohortes chinas (Batista et al., 2020).

- Alopecia Androgenética. Otro estudio informa una observación preliminar de la alta frecuencia de pérdida de cabello de patrón masculino entre los pacientes ingresados con COVID19, y sugiere que la expresión de andrógenos podría ser una pista de la gravedad de COVID-19.

Durante la continua pandemia de SARS-CoV-2 (COVID-19), varios estudios han informado una diferencia significativa en la tasa de casos graves entre mujeres y 9 hombres adultos $(42 \%$ frente a 58\%). Entre niños menores de 14 años, se informó que la tasa de casos graves era extremadamente baja. Para explicar esta diferencia, se han propuesto varias teorías, incluidos el hábito de fumar y el estilo de vida. Sin embargo, ninguna teoría se ajusta tanto a la diferencia de género en casos graves como a la reducción del riesgo en niños prepúberes (Goren et al., 2020).

\section{Conclusiones}

De los resultados mostrados, de su análisis y de su discusión, se pueden obtener las siguientes conclusiones, sobre la COVID-19 en Iberoamérica y la dermatología: 1) Conocer las fuentes de información de los pacientes y los médicos ante una pandemia puede ser de utilidad para mejorar las estrategias de comunicación.; 2) A medida que avancen las diferentes investigaciones podremos establecer con claridad los mecanismos fisiopatológicos relacionadas con las manifestaciones diversas observadas en piel y a los hallazgos histopatológicos de las mismas.; y 
3) El estudio ampliado de la patología cutánea en COVID-19, podría ser una herramienta fundamental para el diagnóstico y tratamiento.

\section{Referencias}

Batista, O. A., Perera, J. C. H., \& Aguilera, K. Y. V. (2020). Dengue y COVID-19: Una aleación amenazadora para las Américas. Lancet, 20, 536.

Borges, M., Borges, M., Borges, J., \& Bastidas, R. (2020). Estudio experimental: manejo del metisoprinol en pacientes con COVID-19. Universidad Ciencia y Tecnología, 24(103), 41-50.

Calvache, N., Jaimes, J. C., Candelo, P., Prada, C., Pineda, Y., Peña, E., \& Fierro, E. (2020). Perspectiva dermatología y COVID-19. Revista de la Asociación Colombiana de Dermatología y Cirugía Dermatol 28(1), 17-26.

Calvo, C., López-Hortelano, M. G., de Carlos Vicente, J. C., Martínez, J. L. V., Ramos, J. T., ... \& Fumadó, V. (2020, March). Recomendaciones sobre el manejo clínico de la infección por el «nuevo coronavirus» SARSCoV2. Grupo de trabajo de la Asociación Española de Pediatría (AEP). In Anales de Pediatría. Elsevier Doyma.

Cañete R., Noda Albelo, A. L., Ferreira Moreno, V., Brito Pérez, K., \& García Herrera, A. L. (2020). SARS-Cov-2, el virus emergente que causa la pandemia de COVID-19. Revista Médica Electrónica, 42(3), 1862-1881.

Carrascosa, J. M., Morillas, V., Bielsa, I., \& Munera-Campos, M. (2020). Manifestaciones cutáneas en el contexto de la infección por SARS-CoV-2 (COVID-19). Actas Dermo-Sifiliográficas.

Echeverría, R. R., Caceres, O. J., Quiñones-Laveriano, D. M., \& Sueyoshi, J. H. (2020). Manifestaciones cutáneas en un paciente con COVID-19. Reporte de un caso en la selva peruana. Revista de la Facultad de Medicina, 69(1).

Fernández Bosch, A., Martínez de la Torre, C., Córcoles García, S., \& Serna Serrano, B. (2020). Manifestaciones cutáneas por COVID-19 en paciente polisintomática. Revista Clínica de Medicina de Familia, 13(3), $235-239$.

Giuseppe, G., Terán, C. G., Martínez, A., \& Volz, A. (2020). Covid-19, una mirada desde la pediatría. Gaceta Médica Boliviana, 43(1), 56-66.

Goren, A., McCoy, J., Wambier, CG, Vano que ver la alopecia androgenética con COVID-19? Una idea de una posible nueva terapia. Terapia dermatológica

Granados Campos, L., Broche del Pino, L., Pérez Leal, L., \& López Rodríguez, V. M. (2020). Manifestaciones cutáneas en pacientes pediátricos infectados por el coronavirus SARS-CoV-2. Revista Cubana de Pediatría, 92.

Herrera, J., Peñafiel, A., \& Rivas, M. Manifestaciones cutáneas en la infección de COVID 19: Revisión Bibliográfica.

Macias, M., Zapata, E., González, M., Fuenzalida, H., Honeyman, J., Chávez, F., ... \& Albornoz, R. (2020). Recomendaciones Rama de Dermatología Pediátrica, Sociedad Chilena de Pediatría. Pandemia COVID-19. Revista chilena de pediatría, 91(7), 43-48.

Maguiña Vargas, C., Gastelo Acosta, R., \& Tequen Bernilla, A. (2020). El nuevo Coronavirus y la pandemia del Covid-19. Revista Medica Herediana, 31(2), 125-131.

Martinez Torres, E., \& Sabatier García, J. (2020). Dengue y COVID-19: semejanzas y diferencias. Revista Cubana de Pediatría, 92.

IPSA SCIENTIA - Vol. 5 No $1-2020$ 
Martin-Gorgojo, A., Bernabeu-Wittel, J., Linares-Barrios, M., Russo-de la Torre, F., García-Doval, I., \& del Río-de la Torre, E. (2020). Congreso virtual de dermatología realizado a través de Telegram ${ }^{\circledR}$ durante el confinamiento de la COVID-19: organización y evaluación. Actas Dermo-Sifiliográficas.

Monte Serrano, J., García-Gil, M. F., Matovelle Ochoa, C., \& Cruañes Monferrer, J. (2020). Lesiones cutáneas tipo perniosis durante la epidemia COVID-19 (No. ART-2020-118253).

Mora, A. S., Castillo, A. S., \& Ellis, C. G. (2020). SARS-CoV-2: la nueva pandemia. Revista Medica Sinergia, 5(7), e538-e538.

Morey-Olivé, M., Espiau, M., Mercadal-Hally, M., Lera-Carballo, E., \& García-Patos, V. (2020, June). Manifestaciones cutáneas en contexto del brote actual de enfermedad por coronavirus 2019. In Anales De Pediatria (Barcelona, Spain: 2003) (Vol. 92, No. 6, p. 374). Elsevier.

Oña, M. A., Oña, C. G., Moreta, E. A., \& Serrano, H. L. O. (2020). Serie de Casos de Sars-cov-2: Aspectos y Características de la Enfermedad Covid-19. Revista Científica Hallazgos21, 5(3), 380-390.

Osuna, B. G., Romero, P. M. A., \& Ozuna, M. J. G. (2020). El coronavirus y la salud como derecho humano a de las TIC: Ecuador y Brasil. Revista Ibérica de Sistemas e Tecnologias de Informação, (E32), 387-399.

Padilla Machaca, P. M., Cárdenas Ramírez, B. E., \& Cabrera Cabrejos, M. C. (2020). Impacto del COVID-19 en las enfermedades hepáticas y la salud pública en el Perú. Revista de Gastroenterología del Perú, 40(2), 162-172.

Pérez, M. R., Gómez Tejeda, J. J., \& Dieguez Guach, R. A. (2020). Características clínico-epidemiológicas de la COVID-19. Revista Habanera de Ciencias Médicas, 19(2).

Pérez-García, U., \& Arellano-Mendoza, M. I. (2020). Labor de Dermatología en la atención a reacciones cutáneas adversas por uso de equipo de protección personal durante la pandemia por COVID-19 Dermatology work in attention to adverse skin reactions due to the use of personal protective equipment during the COVID-19 pandemic. Dermatología Revista Mexicana, 64(4), 487-490.

Rebollar, J. C. O. (2020). Manifestaciones cutáneas de la infección por COVID-19. A propósito de un caso. Medicina Clinica, 155(1), 50.

Recalcati S. (2020). Cutaneous Manifestations in CO- VID-19: A First Perspective. J. Eur. Acad. Dermatol. Venereol. 2020 Mar 26 doi.org/10.1111/jdv.16387

Romaní, J., Baselga, E., Mitjà, O., Riera-Martí, N., Garbayo, P., Vicente, A., ... \& Calzado, S. (2020). Lesiones pernióticas y acrales en España durante el confinamiento por COVID: análisis retrospectivo de 12 casos. Actas Dermo-Sifiliográficas.

Salas-Asencios, R., Iannacone-Oliver, J., Guillén-Oneeglio, A., Tantaléan-Da Fieno, J., Alvariño-Flores, L., Castañeda-Pérez, L., \& de León, L. C. P. (2020). Coronavirus Covid-19: Conociendo Al Causante De La Pandemia. The Biologist, 18(1).

Serrano, J. M., Monferrer, J. C., \& García-Gil, M. F. (2020). Lesiones cutáneas acro-isquémicas durante la epidemia COVID-19. Semergen.

Tapia, E. J. G., López, A. M. M., Reyez, M. D. L. Á. S., \& Ramírez, G. R. (2020). Características clínicoepidemiológicas y algunas reflexiones sobre la COVID-19 en Holguín, Cuba, 2020. Correo Científico Médico, 24(3).

Veintimilla-Quintana, P., Pazmiño, M. B., \& Coral-Rosero, A. P. (2020). Síntomas no respiratorios del COVID-19. Urticaria en pacientes asintomáticos. Dermatología Revista Mexicana, 64(3), 287-293.

IPSA SCIENTIA - Vol. 5 No $1-2020$ 
Zimman, S., Cura, M. J., Luna, P. C., Echeverría, C. M., \& Mazzuoccolo, L. D. (2020). Impacto de la pandemia COVID-19 en los tratamientos inmunomoduladores e inmunosupresores en dermatología: actitudes de los pacientes y los dermatólogos en Argentina. Actas dermo-sifiliogr.(Ed. impr.), 0-0. 